\title{
Combining Null-Steering and Adaptive Filtering for Acoustic Feedback Cancellation in a Multi-Microphone Earpiece
}

\author{
Henning Schepker*, Linh T. T. Tran ${ }^{\dagger}$, Sven Nordholm ${ }^{\dagger}$ Simon Doclo* \\ * Department of Medical Physics and Acoustics and Cluster of Excellence Hearing4All, \\ University of Oldenburg, Germany \\ \{henning.schepker,simon.doclo\}@ uni-oldenburg.de \\ $\dagger$ Department of Electrical and Computer Engineering, Curtin University, Bentley, WA, Australia \\ t.tran57@postgrad.curtin.edu.au, s.nordholm@curtin.edu.au
}

\begin{abstract}
Commonly adaptive filters are used to reduce the acoustic feedback in hearing aids. While theoretically allowing for perfect cancellation of the feedback signal, in practice the adaptive filter solution is typically biased due to the closedloop hearing aid system. In contrast to conventional behindthe-ear hearing aids, in this paper we consider an earpiece with multiple integrated microphones. For such an earpiece it has previously been proposed to use a fixed null-steering beamformer to reduce the acoustic feedback in the microphones. In this paper we propose to combine the fixed null-steering beamformer with an additional adaptive filter to cancel the residual feedback component in the beamformer output. We compare the combination of the fixed null-steering beamformer and different adaptive filtering algorithms including subband adaptive filtering and the prediction-error-method based fullband adaptive filtering with using either of the two approaches alone. Experimental results using measured acoustic feedback show the benefit of using the combined approach compared to using either of the two approaches to cancel the acoustic feedback.
\end{abstract}

\section{INTRODUCTION}

Acoustic feedback occurs due to coupling between the hearing aid loudspeaker and the microphone(s) and is a common problem in hearing aids that limits the maximum applicable gain. In addition, the acoustic feedback can degrade the sound quality and is often perceived as whistling or howling. Therefore, in order to increase the maximum gain and increase the sound quality in a hearing aid, robust feedback cancellation algorithms are needed.

Frequently adaptive feedback cancellation schemes are used to cancel the acoustic feedback, which use an adaptive filter to model the acoustic feedback path(s) between the hearing aid loudspeaker and the microphone(s) [1], [2], [3], [4], [5], [6]. Theoretically these approaches allow for perfect cancellation of the feedback signal. However, due to the closed-loop system of the hearing aid, the filter adaptation is usually biased [7], [8]. In order to reduce this bias several solutions have been proposed for single-loudspeaker single-microphone hearing aids including noise injection [3], adaptive prewhitening [8], and phase modulation [9]. Furthermore, several approaches to improve the feedback cancellation performance using multiple

This work was supported in part by the Research Unit FOR 1732 "Individualized Hearing Acoustics" and the Cluster of Excellence 1077 "Hearing4All", funded by the German Research Foundation (DFG) and project 57142981 "Individualized acoustic feedback cancellation" funded by the German Academic Exchange Service (DAAD).

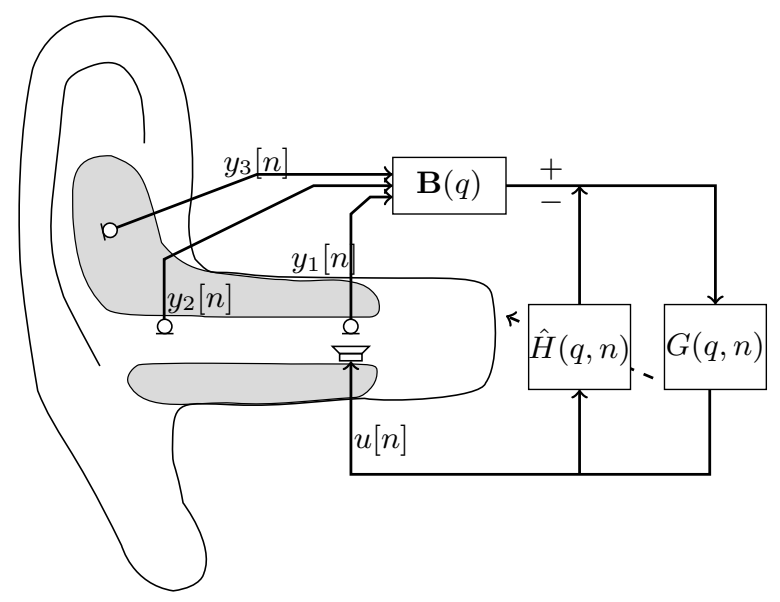

Fig. 1. Hearing aid system considered in this work.

microphones have been proposed, e.g., by adaptively reducing the contribution of the incoming signal on the adaptive filter update [5], using a fixed null-steering beamformer [10], [11] or by using a combined multi-microphone feedback cancellation and noise reduction scheme [12], [13].

In this paper we consider the combination of a fixed null-steering beamformer to cancel the contribution of the loudspeaker signal in the microphone(s) with an adaptive filter aiming to cancel the residual feedback component in the beamformer output for a newly developed earpiece [14]. Figure 1 depicts the design of the earpiece with three microphones and a single loudspeaker, where two microphones and the loudspeaker a closely spaced in the vent and the third microphone is located in the concha. Based on this physical design a robust beamformer can be designed that steers a spatial null in the direction of the hearing aid loudspeaker located in the vent [11], [15]. Ideally the beamformer alone cancels all signals originating from the inside of the ear canal while not (largely) impacting the incoming signal. However, changes in the acoustic feedback path may limit the performance of the fixed null-steering beamformer. Therefore, we propose to additionally use an adaptive filter to cancel the residual feedback component.

Different adaptive filtering algorithms have been proposed 


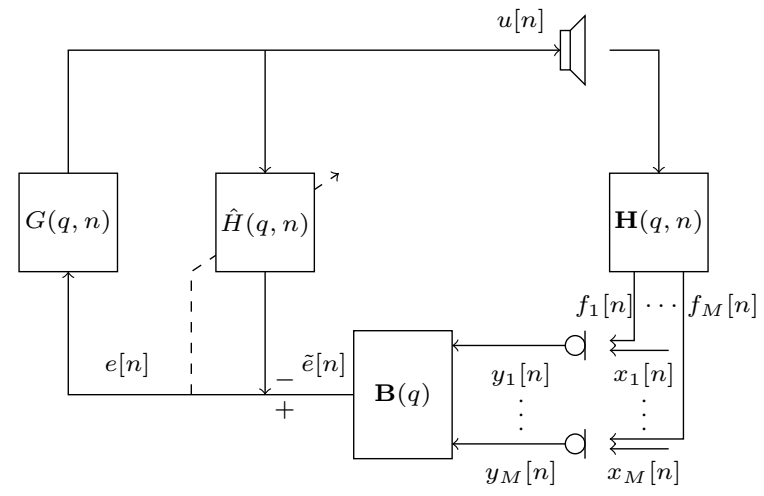

Fig. 2. Single-loudspeaker multi-microphone hearing aid system considered in this paper.

for adaptive feedback cancellation, including fullband [8], [16], [17], subband [4], [18], and frequency-domain algorithms [12], [19], [20]. In this paper we compare the performance of the combination of a robust null-steering beamformer with two different adaptive filtering algorithms: a) the fullband normalized least-mean squares (NLMS) using the predictionerror-method (PEM) [8] for decorrelation and b) the subband NLMS adaptive filter in a delayless structure [22]. Experimental results using measured acoustic feedback path show that using the proposed combination of a null-steering beamformer and an adaptive filter yields an improvement of approximately $15 \mathrm{~dB}$ in added stable gain compared to using only an adaptive filter.

\section{Acoustic Scenario And Notation}

Consider the acoustic system of a single-loudspeaker multimicrophone hearing aid with $M$ microphones depicted in Figure 2. The microphone signal $y_{m}[n], m=1, \ldots, M$, in the $m$ th microphone at discrete time $n$ is the sum of the incoming signal $x_{m}[n]$ and the feedback contribution $f_{m}[n]$ of the loudspeaker in the $m$ th microphone and is denoted using matrix and vector notation as

$$
\mathbf{y}[n]=\mathbf{x}[n]+\underbrace{\mathbf{H}(q, n) u[n]}_{\mathbf{f}[n]},
$$

with

$$
\begin{aligned}
\mathbf{y}[n] & =\left[\begin{array}{lll}
y_{1}[n] & \ldots & y_{M}[n]
\end{array}\right]^{T}, \\
\mathbf{x}[n] & =\left[\begin{array}{lll}
x_{1}[n] & \ldots & x_{M}[n]
\end{array}\right]^{T}, \\
\mathbf{H}(q, n) & =\left[\begin{array}{lll}
H_{1}(q, n) & \ldots & H_{M}(q, n)
\end{array}\right]^{T},
\end{aligned}
$$

where $[\cdot]^{T}$ denotes transpose operation, $u[n]$ is the loudspeaker signal, and $H_{m}(q, n)$ is the transfer function of the acoustic feedback path between the $m$ th microphone and the loudspeaker. We assume that the acoustic feedback path can be modeled as an $L_{H}$-dimensional polynomial in $q$, i.e.,

$$
\begin{aligned}
H_{m}(q, n) & =h_{m, 0}[n]+\cdots+h_{m, L_{H}-1}[n] q^{-L_{H}+1} \\
& =\mathbf{h}_{m}^{T}[n] \mathbf{q},
\end{aligned}
$$

where $\mathbf{q}$ is the vector containing the delay-elements of $q$ of appropriate length and $\mathbf{h}_{m}[n]=\left[\begin{array}{lll}h_{m, 0}[n] & \ldots & h_{m, L_{H}-1}[n]\end{array}\right]^{T}$ is the $L_{H}$-dimensional vector of the impulse response of the $m$ th acoustic feedback path.

After applying the fixed filter-and-sum beamformer $\mathbf{B}(q)$ to the microphone signals the beamformer output signal $\tilde{e}[n]$ is obtained, i.e.,

$$
\tilde{e}[n]=\mathbf{B}^{T}(q) \mathbf{y}[n],
$$

with

$$
\mathbf{B}(q)=\left[\begin{array}{lll}
B_{1}(q) & \ldots & B_{M}(q)
\end{array}\right]^{T} .
$$

The $L_{B}$-dimensional beamformer coefficient vector for the $m$ th microphone is defined as

$$
\mathbf{b}_{m}=\left[\begin{array}{lll}
b_{m, 0} & \ldots & b_{m, L_{B}-1}
\end{array}\right]^{T},
$$

and the $M L_{B}$-dimensional stacked vector is defined as

$$
\mathbf{b}=\left[\begin{array}{lll}
\mathbf{b}_{1}^{T} & \ldots & \mathbf{b}_{M}^{T}
\end{array}\right]^{T} .
$$

Furthermore, an adaptive filter $\hat{H}(q, n)$ of length $L_{\hat{H}}$ is used to cancel the (remaining) contribution of the acoustic feedback signals in the beamformer output resulting in the error signal $e[n]$, i.e.,

$$
e[n]=\tilde{e}[n]-\hat{H}(q, n) u[n] .
$$

The signal $e[n]$ is then processed using the hearing aid forward path $G(q, n)$, yielding the loudspeaker signal $u[n]$, i.e.,

$$
u[n]=G(q, n) e[n] .
$$

\section{SyStEM ANALYSIS}

We first analyze the transfer function of the hearing aid system depicted in Figure 2 and present conditions under which perfect feedback cancellation can be achieved. Using (1), (7), (11), and (12) loudspeaker signal can be rewritten as

$$
\begin{aligned}
u[n]= & G(q, n)\left(\mathbf{B}^{T}(q) \mathbf{y}[n]-\hat{H}(q, n) u[n]\right) \\
= & G(q, n) \mathbf{B}^{T}(q) \mathbf{x}[n] \\
& +G(q, n)\left(\mathbf{B}^{T}(q) \mathbf{H}(q, n)-\hat{H}(q, n)\right) u[n],
\end{aligned}
$$

such that

$$
u[n]=\underbrace{\frac{G(q, n) \mathbf{B}^{T}(q)}{1-G(q, n)\left(\mathbf{B}^{T}(q) \mathbf{H}(q, n)-\hat{H}(q, n)\right)}}_{\mathbf{C}^{T}(q, n)} \mathbf{x}[n],
$$

with $\mathbf{C}(q, n)$ the closed-loop transfer function. From this expression it can be observed that perfect feedback cancellation for the considered system can be achieved under either of the following two conditions:

- the null-steering beamformer $\mathbf{B}(q)$ and the adaptive filter $\hat{H}(q, n)$ cancel the feedback contribution in the microphones, i.e.,

$$
\mathbf{B}^{T}(q) \mathbf{H}(q, n)-\hat{H}(q, n)=0 .
$$

- the adaptive filter is not used, i.e., $\hat{H}(q, n)=0$, and the null-steering beamformer $\mathbf{B}(q)$ cancels the feedback contribution in the microphones, i.e.,

$$
\mathbf{B}^{T}(q) \mathbf{H}(q, n)=0,
$$

with $B_{m}(q) \neq 0$ for at least one $m \in[1, \ldots, M]$ to avoid the trivial solution. 


\section{PRoposed COMBined NulL-STEERING AND ADAPTIVE FILTERING}

Since the second condition for perfect feedback cancellation (cf. Section III) is difficult to fulfill in general, in this paper we propose to use the combination of a fixed null-steering beamformer and an adaptive feedback canceller aiming to fullfil the first condition for perfect feedback cancellation. We first review the computation of the null-steering beamformer as presented in [11] and then present two different adaptive filtering algorithms that are used for adaptive feedback cancellation in the proposed system. For the proposed system these aim to reduce the residual feedback component in the nullsteering beamformer output.

\section{A. Null-steering beamformer design}

The goal of the null-steering beamformer is to cancel the contribution of the loudspeaker signal in the microphone(s). Assuming time-invariance and knowledge of a set of multiple (I) acoustic feedback paths, e.g., by measurement, the nullsteering beamformer is designed by minimizing the following constrained least-squares optimization problem [11]

$$
\begin{array}{cl}
\min _{\mathbf{b}} & \sum_{i=1}^{I}\left\|\mathbf{H}_{i}^{T} \mathbf{b}\right\|_{2}^{2} \\
\text { subject to } & B_{m_{0}}(q)=q^{-L_{D}},
\end{array}
$$

where in the beamformer weights in the reference microphone $m_{0}$ are constrained to be a simple delay of $L_{D}$ samples. Furthermore, $\mathbf{H}_{i}^{T}$ is the $\left(L_{B}+L_{H}-1\right) \times M L_{B}$-dimensional matrix of the stacked $\left(L_{B}+L_{H}-1\right) \times L_{B}$-dimensional convolution matrices $\mathbf{H}_{m, i}^{T}$ of the $i$ th measurement in the $m$ th microphone. Note that for this optimization problem a closedform solution exists and the reader is refered to [11] for more details.

\section{B. Adaptive Feedback Cancellation}

The goal of the adaptive filter $\hat{H}(q, n)$ is to remove the residual feedback component in the beamformer output signal $\tilde{e}[n]$. In order to update the adaptive filter coefficients $\hat{\mathbf{h}}[n]$ we use two different established algorithms. First we consider the fullband adaptation and use the PEM to reduce the bias [8] and second we use a delayless subband adaptation of the filter coefficients which has been shown to work well for speech signals [18].

1) Prediction-Error-Method: One possibility to decorrelate the loudspeaker signal and the incoming signal in the filter adaptation is to use the PEM. Assuming that the incoming signal in the beamformer output $\tilde{x}[n]=\mathbf{B}^{T}(q) \mathbf{x}[n]$ can be modeled as an autoregressive process with a white Gaussian noise excitation signal $w[n]$, i.e.,

$$
\tilde{x}[n]=A^{-1}(q, n) w[n],
$$

with $A^{-1}(q, n)$ a monic and stable all-pole filter of order $L_{A}$, the goal of the PEM is to first obtain an estimate $\hat{A}(q, n)$ of $A(q, n)$ in order to prewhiten the beamformer output $\tilde{e}[n]$ and the loudspeaker signal $u[n]$. By using $\hat{A}(q, n)$ the decorrelated signals $\tilde{e}_{p}[n]=\hat{A}(q, n) \tilde{e}[n]$ and $u_{p}[n]=\hat{A}(q, n) u[n]$ are computed and used in the filter adaptation, where the prewhitened a-priori error signal is defined as

$$
e_{p}[n]=\tilde{e}_{p}[n]-\hat{H}(q, n-1) u_{p}[n] .
$$

In the proposed approach $\hat{A}(q, n)$ is computed using the Levinson-Durbin recursion [21] every $10 \mathrm{~ms}$ from the most recent $10 \mathrm{~ms}$ of the error signal $e[n]$, similar as proposed in [19]. The adaptive filter $\hat{H}(q, n)$ is then updated in the timedomain using the NLMS [21] update rule, i.e.,

$$
\hat{\mathbf{h}}[n]=\hat{\mathbf{h}}[n-1]+\frac{\mu}{\alpha+\mathbf{u}_{p}^{T}[n] \mathbf{u}_{p}[n]} \mathbf{u}_{p}[n] e_{p}[n],
$$

where $\mathbf{u}_{p}[n]$ is the $L_{\hat{H}}$-dimensional vector of the prewhitened loudspeaker signal, $\mu$ is a step-size, and $\alpha$ is a small positive constant.

2) Delayless subband adaptive filter: A different possibility to decorrelate the loudspeaker signal and the incoming signal is to use subband adaptive filtering algorithm. Recently it has been shown that this approach works particularly well for speech signal without any additional decorrelation units [18]. In this paper we therefore also consider the use of a delayless subband adaptive filter architecture in closed-loop configuration [22] with $M_{s}$ subbands and a decimation factor of $D_{s}=M_{s} / 2$. The error signal $e[n]$ and $u[n]$ are transformed into the subband domain using an analysis filterbank based on a Kaiser prototype window [23]. In each subband an adaptive filter of length $L_{s}=L_{\hat{H}} / D$ is updated using the NLMS update rule similar as in the fullband adaptive filter (cf. (21)). The corresponding fullband filter coefficients are then obtained by using the weight transform scheme presented in [24].

\section{EXPERIMENTAL EVALUATION}

The performance of the proposed combined null-steering and adaptive filtering algorithms for acoustic feedback cancellation is evaluated and compared with using only the nullsteering beamformer and with using only an adaptive filtering algorithm. Particularly we consider the ability to cancel the acoustic feedback in a challenging acoustic scenarios with varying broadband gains.

\section{A. Setup and Performance Measures}

We used measured acoustic feedback paths from the threemicrophone earpiece depicted in Figure 1. Measurements were performed on a dummy head with adjustable ear canals [25] in a an acoustically treated chamber. The impulse responses of the acoustic feedback paths were sampled at $f_{s}=16 \mathrm{kHz}$ and truncated to length $L_{H}=100$. In total 20 different sets of acoustic feedback paths were measured, i.e., the earpiece was repositioned on the dummy head 10 times and for each repositioning feedback paths were measured in both free-field, i.e., without obstruction, and with a telephone receiver in close distance to the ear. For the forward path $G(q, n)=|G| q^{-d_{G}}$ of the hearing aid we always used a delay $d_{G}=96$, corresponding $6 \mathrm{~ms}$, while different broadband amplifications $|G|$ were used. For all experiments we used $M=2$ microphones ( $m=1,2)$ and the reference microphone $m_{0}=2$, i.e., the microphone located at the outer end of the vent, was chosen as it provides a natural position for sound pickup likely including 
all relevant perceptual cues. The null-steering beamformer was optimized using 9 out of the 10 free-field measurements with $L_{B}=16$ coefficients for each microphone and a delay of $L_{D}=L_{B} / 2$ in the reference microphone.

The parameters of the PEM-based fullband adaptive filter (FB-PEM) were chosen as $L_{\hat{H}}=64, \mu=0.002$ and $\alpha=10^{-6}$. The order of the prediction error was chosen as $L_{A}=20$. The parameters of the subband adaptive filter (SB) were chosen as $M_{s}=32, D_{s}=16, L_{s}=4$, $\mu=0.003, \alpha=10^{-6}$ and an 128-point DFT was used to obtain the corresponding fullband filter. The parameters of both adaptive filtering algorithms were chosen to yield similar initial convergence.

The performance of the proposed combined fixed nullsteering and adaptive filtering approach was evaluated using the added stable gain (ASG) [8] and the effective closed-loop gain (ECLG) [4]. The $A S G$ for the considered hearing aid system is computed as [8]

$$
A S G=20 \log _{10} \mathcal{M}-20 \log _{10} \frac{1}{\max _{f}\left|H_{m_{0}}(f)\right|},
$$

where the second term is the maximum stable gain in the reference microphone $m_{0}$ without applying the beamformer and/or adaptive filter and $\mathcal{M}$ is in the maximum stable gain using the proposed approach, i.e.,

$$
\mathcal{M}=\frac{1}{\max _{f}\left|\mathbf{H}^{H}(f) \mathbf{B}(f)-\hat{H}(f)\right|},
$$

where $\mathbf{H}(f)$ is the stacked vector of the frequency responses at frequency $f$ of each microphone, $\mathbf{B}(f)$ is the stacked vector of the frequency responses of the beamformer in each microphone and $\hat{H}(f)$ is the frequency response of the adaptive filter.

The ECLG for a broadband gain $|G|$ is computed as

$$
E C L G=20 \log _{10}|G|-20 \log _{10} \mathcal{M} .
$$

Whenever the ECLG exceeds a value of $0 \mathrm{~dB}$ the system can be assumed to be howling [4].

As the incoming signal we used an 80 s long speech signal comprising 26 concatenated sentences spoken by 4 different speakers from the TIMIT database [26]. The distance between the external source and the dummy head was $1.2 \mathrm{~m}$.

\section{B. Results}

In the experiment we investigate the performance of all considered combinations of the null-steering beamformer and adaptive filtering algorithms for a time-varying broadband gain. Figure 3 depicts the results, where the red line indicates the time-course of the overcritical gain, where $G_{0}$ was chosen to result in an overcritical gain of approximately $25 \mathrm{~dB}$. As can be observed all considered systems perform very similar. Note that the null-steering beamformer alone yields an unstable system after $10 \mathrm{~s}$ by the choice of the overcritical gain and is thus not shown.

In order to allow for an easier comparison for different overcritical gains, in the following we consider the ECLG. We compute the ECLG for the last $10 \mathrm{~s}$ of the speech signal where the highest gain is applied for different broadband gains. Figure 4 depicts the distributions of the ECLG by

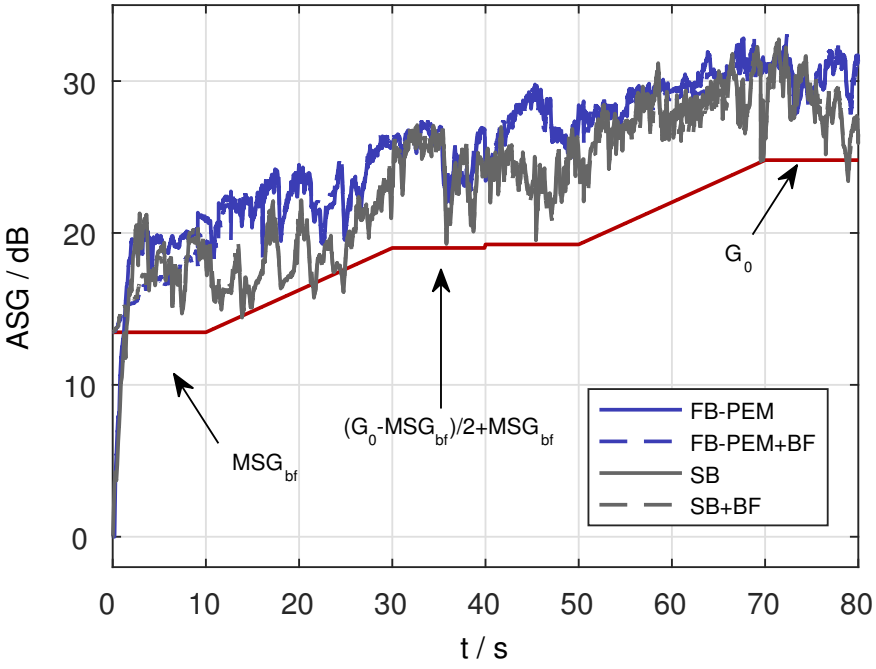

Fig. 3. Exemplary ASG results for a forward path gain of $G_{0}$ that is approximately $25 \mathrm{~dB}$ overcritical. The red line indicates the time-varying gain and $\mathrm{MSG}_{\mathrm{bf}}$ denotes the maximum stable gain of using the null-steering beamformer alone. Note that the change of the acoustic feedback paths after $40 \mathrm{~s}$ is small due to the physical design of the earpiece.

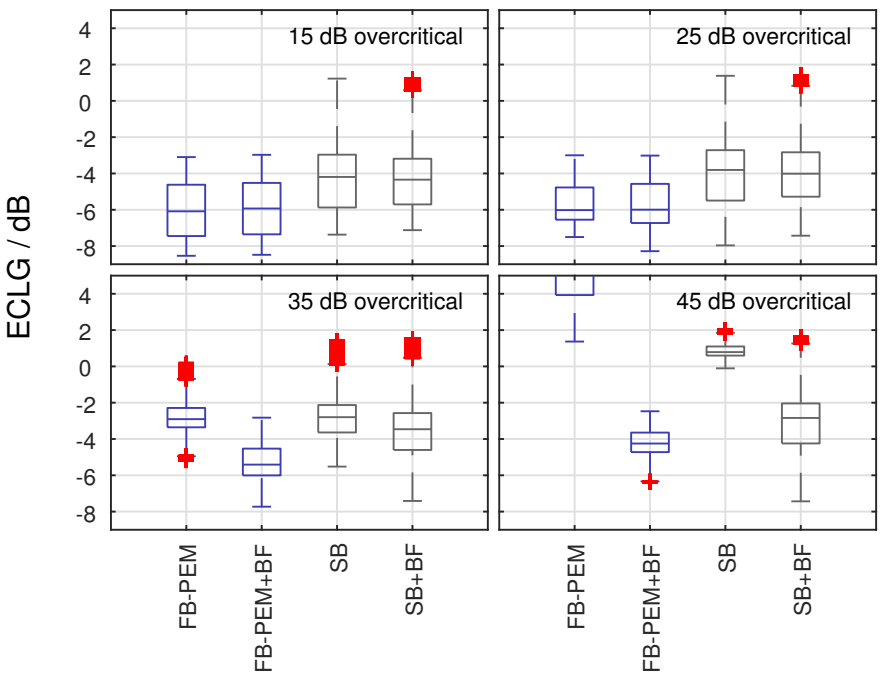

Fig. 4. Exemplary ECLG results for different overcritical gains.

means of boxplots for four different overcritical gains. As can be observed for lower gains all system lead to a stable performance (i.e., ECLG $<0$ ), where the system using the FB-PEM shows slightly better performance compared to the subband adaptive filtering. For the largest considered gain of $45 \mathrm{~dB}$ overcritical only the combination with the null-steering beamformer leads to a stable system for both adaptive filtering algorithms.

To evaluate the additional benefit of the null-steering beamformer in comparison to using only an adaptive filter, we compute the median ECLG for different overcritical gains for the FB-PEM and FB-PEM+BF systems. Figure 5 depicts the median ECLG as a function of the overcritical gain. As can be observed, using only the null-steering beamformer leads to the lowest performance of $\approx 14 \mathrm{~dB}$. When using only the FB- 


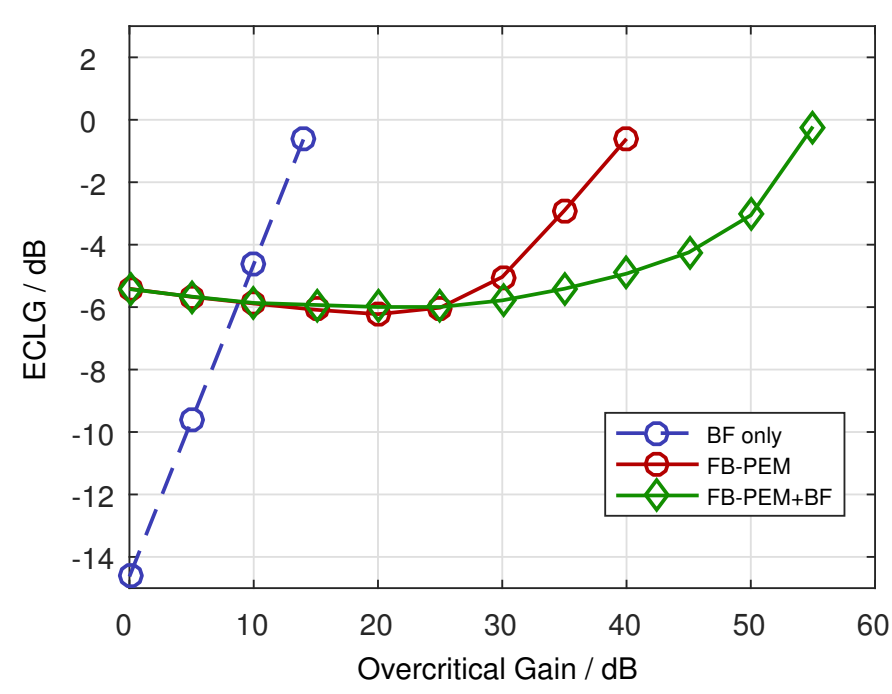

Fig. 5. ECLG as a function of the overcritical gain for the null-steering beamformer only, the FB-PEM only, and the FB-PEM+BF combination.

PEM, the system remains stable for overcritical gains of up to $40 \mathrm{~dB}$, while the combination of the null-steering beamformer and the adaptive filter remains stable for overcritical gains of up to $55 \mathrm{~dB}$. Thus the null-steering beamformer adds an additional $15 \mathrm{~dB}$ in ASG to the system, which is approximately the same amount that could be achieved by using the nullsteering beamformer only. Note that this is also observed when using the subband adaptive filter.

\section{CONCLUSION}

In this paper we propose to combine a null-steering beamformer and an adaptive filter to cancel the acoustic feedback for a multi-microphone earpiece. We compared different adaptive filtering algorithms to cancel the residual feedback component in the beamformer output. Experimental results using measured acoustic feedback paths show that the proposed combination yields an increased performance compared to using only the null-steering beamformer or using only an adaptive filter, where no major differences between the two considered adaptive filtering algorithms were found. In fact, the results indicate that the performance of the combined system is approximately $15 \mathrm{~dB}$ larger than using an adaptive filter only, which can be related to the performance of the null-steering beamformer.

\section{REFERENCES}

[1] T. van Waterschoot and M. Moonen, "Fifty Years of Acoustic Feedback Control: State of the Art and Future Challenges," Proc. IEEE, vol. 99, no. 2, pp. 288-327, Feb. 2011.

[2] A. Spriet, S. Doclo, M. Moonen, and J. Wouters, "Feedback Control in Hearing Aids," in Springer Handbook of Speech Processing, pp. 979-999. Springer-Verlag, Berlin, Germany, 2008.

[3] M. Guo, S. H. Jensen, and J. Jensen, "Novel Acoustic Feedback Cancellation Approaches in Hearing Aid Applications Using Probe Noise and Probe Noise Enhancement," IEEE Trans. Audio, Speech, Language Process., vol. 20, no. 9, pp. 2549-2563, Nov. 2012.

[4] F. Strasser, H. Puder, "Adaptive feedback cancellation for realistic hearing aid applications," IEEE Trans. Audio, Speech, Lang. Process., vol. 23, no. 12, pp. 2322-2333, Dec. 2015.

[5] C. R. C. Nakagawa, S. Nordholm, and W.-Y. Yan, "Analysis of Two Microphone Method for Feedback Cancellation," IEEE Signal Process. Lett., vol. 22, no. 1, pp. 35-39, Jan. 2015.
[6] H. Schepker, L. T. T. Tran, S. Nordholm, S. Doclo, "Improving adaptive feedback cancellation in hearing aids using an affine combination of filters," in Proc. Int. Conf. Acoust. Speech Signal Process. (ICASSP), Shanghai, China, Mar. 2016, pp. 231-235.

[7] M. G. Siqueira and A. Alwan, "Steady-state analysis of continuous adaptation in acoustic feedback reduction systems for hearing-aids," IEEE Trans. Speech Audio Process., vol. 8, no. 4, pp. 443-453, July 2000.

[8] A. Spriet, I. Proudler, M. Moonen, and J. Wouters, "Adaptive feedback cancellation in hearing aids with linear prediction of the desired signal," IEEE Trans. Signal Process., vol. 53, no. 10, pp. 3749-3763, Oct. 2005.

[9] M. Guo, S. H. Jensen, J. Jensen, and S. L. Grant, "On the use of a phase modulation method for decorrelation in acoustic feedback cancellation," in Proc. Europ. Signal Process. Conf. (EUSIPCO), Bucharest, Romania, Aug. 2012, pp. 2000-2004.

[10] H. Schepker, L. T. T. Tran, S. Nordholm, S. Doclo, "Acoustic feedback cancellation for a multi-microphone earpiece based on a null-steering beamformer," in Proc. Int. Workshop Acoust. Signal Enhance. (IWAENC), Xi'an, China, Sept. 2016, pp. 1-5.

[11] H. Schepker, L. T. T. Tran, S. Nordholm, S. Doclo, "A robust nullsteering beamformer for acoustic feedback cancellation for a multimicrophone earpiece," in Proc. ITG Conf. Speech Communication, Paderborn, Germany, Oct. 2016, pp. 165-169.

[12] A. Spriet, G. Rombouts, M. Moonen, and J. Wouters, "Combined feedback and noise suppression in hearing aids," IEEE Trans. Audio, Speech, Language Process., vol. 15, no. 6, pp. 1777-1790, Aug. 2007.

[13] G. Rombouts, A. Spriet, and M. Moonen, "Generalized sidelobe canceller based combined acoustic feedback- and noise cancellation," Signal Process., vol. 88, no. 3, pp. 571-581, Mar. 2008.

[14] F. Denk, M. Hiipakka, B. Kollmeier, and S. M. A. Ernst, "An individualized acoustically transparent earpiece for hearing devices," Int. J. Audiol., in press.

[15] H. Schepker, L. T. T. Tran, S. Nordholm, S. Doclo, "Null-steering beamformer for acoustic feedback cancellation in a multi-microphone earpiece optimizing the maximum stable gain," in Proc. Int. Conf. Acoust. Speech Signal Process. (ICASSP), New Orleans, USA, Mar. 2017.

[16] L. T. T. Tran, H. H. Dam, S. E. Nordholm, "Affine projection algorithm for acoustic feedback cancellation using prediction error method in hearing aids," in Proc. Int. Workshop Acoust. Signal Enhance. (IWAENC), Xi'an, China, Sept. 2016, pp. 1-5.

[17] L. T. T. Tran, H. Schepker, S. Doclo, H. H. Dam, S. E. Nordholm, "Proportionate NLMS for adaptive feedback cancellation in hearing aids," in Proc. Int. Conf. Acoust. Speech Signal Process. (ICASSP), New Orleans, USA, Mar. 2017.

[18] M. Guo, S. H. Jensen, J. Jensen, "Evaluation of State-of-the-Art Acoustic Feedback Cancellation Systems for Hearing Aids," J. Audio Eng. Soc., vol. 61, no. 3, pp. 125-137, Mar. 2013.

[19] A. Spriet, G. Rombouts, M. Moonen, J. Wouters, "Adaptive feedback cancellation in hearing aids," J. Franklin Inst., vol. 343, no., pp. 545-573, Sept. 2006.

[20] G. Bernardi, T. van Waterschoot, J. Wouters, M. Hillbratt, M. Moonen, "A PEM-based frequency-domain Kalman filter for adaptive feedback cancellation," in Proc. 23th European Signal Process. Conf. (EUSIPCO), Nice, France, Sept. 2015, pp. 270-274.

[21] S. Haykin, Adaptive Filter Theory, 3rd ed. Prentice Hall, 1996.

[22] D. R. Morgan, J. C. Thi, "A delayless subband adaptive filter architecture," IEEE Trans. Signal Process., vol. 43, no. 8, pp. 1819-1830, Aug. 1995.

[23] K. F. C. Yiu, N. Grbic, S. Nordholm, K. L. Teo, ”A hybrid method for the design of oversampled uniform DFT filter banks", Signal Processing, vol. 86., no. 7, pp. 1355-1364, July 2006.

[24] J. Huo, S. Nordholm, Z. Zang, "New weight transform schemes for delayless subband adaptive filtering," in Proc. Global Telecom. Conf. (GLOBECOM), San Antonio, USA, Nov. 2001, pp. 197-201.

[25] M. Hiipakka, M. Tikander, and M. Karjalainen, "Modeling the External Ear Acoustics for Insert Headphone Usage," J. Audio Eng. Soc., vol. 58, no. 4, pp. 269-281, Apr. 2010.

[26] J. S. Garofolo, "Getting started with the darpa timit cd-rom: An acoustic phonetic con- tinuous speech database," Nat. Inst. Standards Technol. (NIST), Gaithersburg, MD, Dec. 1988. 